\title{
BRAGG REFLECTION OF INFRAGRAVITY WAVES BY SANDBARS
}

by

PHILIP L.-F. LIU and YONG-SIK CHO

RESEARCH REPORT NO. CACR-93-07

September, 1993

CENTER FOR APPLIED COASTAL RESEARCH

DEPARTMENT OF CIVIL ENGINEERING

UNIVERSITY OF DELAWARE

NEWARK, DE 19716 


\title{
Bragg Reflection of Infragravity Waves by Sandbars
}

\author{
Philip L.-F. Liu and Yong-Sik Cho \\ School of Civil and Environmental Engineering \\ Cornell University, Ithaca, NY 14853
}

\begin{abstract}
Using a multiple-scale perturbation method we derive a set of governing equations describing the transformation of long wave and short wave components in a wave group. These equations are derived from Boussinesq equations with the assumption that the length scale of wave group modulation is in the same order of magnitude as that of the bottom variation, and is much longer than the length scale for the carrier (short) waves. Therefore the reflection of carrier waves by the topographical variation is small and neglected. Numerical examples are presented to show that long waves associated with wave group can be reflected resonantly by a field of periodic sandbars.
\end{abstract}

\section{Introduction}

Multiple offshore sandbars are common coastal morphological features along the Great Lakes and open coasts (e.g. Short 1975; Katoh 1984). These multiple sandbars appear usually on very mild slope beaches. The number of sandbars varies from 2 
or 3 to as many as 15 and the spacing between two adjacent bars ranges from 10 $\mathrm{m}$ to $500 \mathrm{~m}$, which generally increases offshore. In figure 1 an aerial photograph of multiple offshore bars off the northwestern Danish coast (the nearest city is Soeby) is shown. The largest spacing between two adjacent sandbars shown in the picture is about $250 \mathrm{~m}$ (personal communication with Professor J. Fredsøe).

Much theoretical and experimental research has been performed to determine the possible mechanisms of sandbar formation as well as the influence of sandbars on the wave climate. It has been suggested that the initiation of offshore sandbars is related to mass transport velocity near the seabed under partially reflected waves (e.g. Bagnold 1946; Carter et al. 1973). Carter et al.(1973) also demonstrated experimentally and theoretically, that to initiate the sandbar formulation the minimum reflection coefficient is 0.414 . On a very mild beach with slope of less than 5 per thousand where offshore sandbars usually occurs, the direct wave reflection from the beach is very weak and certainly can not be responsible for the formation of sandbars. Surely other parameters than the reflection coefficient must be decisive. However, strong reflection can occur if the wavelength of incident swells is twice of the wavelength of the sandbars. This mechanism is called Bragg resonance (Davies and Heathershaw 1984; Mei 1985; Hara and Mei 1987; Yoon and Liu 1987; Kirby and Vengayil 1988). Therefore the strong reflection of waves is both the cause and effect of periodic sandbars (Mei and Liu 1993).

For typical sea swells with a period of $12 \mathrm{sec}$, the wavelength of the swell in a 
depth of $10 \mathrm{~m}$ is roughly $120 \mathrm{~m}$. The Bragg resonance suggests that the multiple sandbars should have a spacing of $60 \mathrm{~m}$. Hence the mechanism of Bragg resonance of incident sea swells can not explain the presence of sandbars with much larger spacing such as the ones shown in figure 1.

Infragravity waves with periods of 30 secs to few minutes have been observed in shallow coastal areas and their energy levels are highly correlated with energy in the swell-frequency band (7 to 20 sec periods) (Elgar et al. 1992). Katoh (1984) suggested that while sea swells mobilize the sediments into suspension, the mass transport in the infragravity waves distributes the suspended sediment to form sandbars. Therefore, the interactions between periodic sandbars and infragravity waves may play an important role in the formation of sandbars with large spacings.

Based on the concept of radiation stresses, Hara and Mei (1987) studied the generation of infragravity waves through the interactions of wave packets and periodic sandbars. In their work, the water depth is assumed to be intermediate and the Bragg resonance is associated with the incident carrier (short) waves; i.e. the wavenumber of the sandbars is about twice of that of the incident short waves. Although both bound long waves and free long waves are generated through interactions, they are not resonantly reflected by periodic sandbars.

In this paper, the Bragg scattering of infragravity waves by periodic sandbars is investigated. We focus on the shallow water environment where Boussinesq equations are suitable for describing both sea swells and infragravity waves. For simplicity, 
we assume that the length scale for the sandbar variation is much longer than the wavelength of the sea swells, but is in the same order of magnitude of the length scale for the wave envelope modulation. Therefore, the long waves associated with wave groups might be reflected resonantly, while the reflection of sea swells is small and neglected.

In the following two sections a multiple-scale perturbation approach is used to derive governing equations for each harmonic of sea swells and associated infragravity waves. These equations are derived from the Boussinesq equations and are coupled nonlinear first-order ordinary differential equations. Numerical solutions for the Bragg reflection of infragravity waves by a periodic sandbar are then investigated.

\section{Modulation Equations in Shallow Water}

In this section, a set of modulation equations for groups of sea swells in shallow water is derived from one-dimensional Boussinesq equations. Employing $\omega_{0}$ as the characteristic short wave frequency, $a_{0}$ as the characteristic short wave amplitude and $h_{\circ}$ as the characteristic water depth, we introduce the following dimensionless variables:

$$
\begin{aligned}
& t=\omega_{0} t^{\prime}, \quad x=\frac{\omega_{0}}{\sqrt{g h_{\circ}}} x^{\prime}, \quad h=\frac{1}{h_{0}} h^{\prime} \\
& u=\frac{h_{\circ}}{a_{0} \sqrt{g h_{0}}} u^{\prime}, \quad \zeta=\frac{1}{a_{\circ}} \zeta^{\prime}, \quad \omega=\frac{1}{\omega_{0}} \omega^{\prime}
\end{aligned}
$$

where $\zeta$ is the free-surface displacement, $u$ denotes the depth-averaged horizontal velocity and $g$ is the gravitational acceleration. Using these dimensionless variables, 
Boussinesq equations can be written in the following dimensionless form (Peregrine 1972):

$$
\begin{gathered}
\frac{\partial \zeta}{\partial t}+\frac{\partial}{\partial x}[(h+\epsilon \zeta) u]=O \\
\frac{\partial u}{\partial t}+\epsilon u \frac{\partial u}{\partial x}+\frac{\partial \zeta}{\partial x}=\mu^{2}\left[\frac{1}{2} h \frac{\partial^{2}}{\partial x^{2}}\left(h \frac{\partial u}{\partial t}\right)-\frac{1}{6} h^{2} \frac{\partial^{2}}{\partial x^{2}}\left(\frac{\partial u}{\partial t}\right)\right]+O\left(\epsilon^{2}, \epsilon \mu^{2}, \mu^{4}\right)
\end{gathered}
$$

where

$$
\epsilon=\frac{a_{0}}{h_{\mathrm{o}}} \ll 1, \quad \mu^{2}=\frac{\omega_{0}^{2} h_{0}}{g} \ll 1
$$

$\epsilon$ and $\mu^{2}$ are small parameters representing the nonlinearity and frequency dispersion, respectively. The Boussinesq approximation assumes these two parameters be of the same order of magnitude. We also assume that the variation of depth is small within a typical wavelength, i.e.,

$$
O\left(\left|\frac{d h}{d x}\right|\right) \leq O\left(\epsilon, \mu^{2}\right)
$$

We shall study the propagation and reflection of groups of modulating sea swells with a carrier wave frequency $\omega_{0}$. Because the length and time scales for the wave envelope are longer than those of carrier waves, we introduce slow variables

$$
X=\mu x, \quad T=\mu t
$$

The solutions of equations (2) and (3) can be expressed as:

$$
\zeta(x, t)=\frac{1}{2} \sum_{n} \zeta_{n}(X, T) e^{i n \theta}
$$




$$
u(x, t)=\frac{1}{2} \sum_{n} u_{n}(X, T) e^{i n \theta}
$$

with

$$
\theta=\int \frac{1}{\sqrt{h}} d x-t
$$

in which $n=0, \pm 1, \pm 2, \ldots$ and $\theta$ represents the phase function of a progressive wave propagating in the positive $x$-direction with a local phase speed $\sqrt{h}$. The amplitude functions for the free surface displacement and the velocity, $\zeta_{n}$ and $u_{n}$, modulate slowly in time and space. Substituting equations (6) and (7) into equations (2) and (3) and collecting coefficients of different Fourier components (i.e. n-th harmonic), we obtain a set of continuity and momentum equations for $\zeta_{n}$ and $u_{n}$. Similar approaches have been used by Liu et al. (1985), Yoon and Liu (1987) and Liu et al. (1992).

When $n=0, \zeta_{0}$ represents the combination of a steady-state free surface set-down and long waves under wave groups. The governing equations for $\zeta_{0}$ and $u_{0}$ can be expressed as

$$
\begin{gathered}
\mu \frac{\partial \zeta_{o}}{\partial T}+\mu \frac{\partial}{\partial X}\left(h u_{0}\right)+\frac{\epsilon \mu}{2} \frac{\partial}{\partial X}\left(\zeta_{\circ} u_{o}\right)=-\frac{\epsilon \mu}{2 \sqrt{h}} \sum_{s \neq 0} \frac{\partial}{\partial X}\left(\zeta_{s} \zeta_{-s}\right)+O\left(\epsilon^{2}, \epsilon \mu^{2}, \mu^{4}\right) \\
\mu \frac{\partial u_{o}}{\partial T}+\mu \frac{\partial \zeta_{0}}{\partial X}+\frac{\epsilon \mu}{4} \frac{\partial}{\partial X}\left(u_{\circ} u_{o}\right)=-\frac{\epsilon \mu}{4 h} \sum_{s \neq 0} \frac{\partial}{\partial X}\left(\zeta_{s} \zeta_{-s}\right)+O\left(\epsilon^{2}, \epsilon \mu^{2}, \mu^{4}\right)
\end{gathered}
$$

For $n \neq 0$, we can eliminate $u_{n}$ from the resulting continuity and momentum equations to get a set of equations for $\zeta_{n}$.

$$
\mu^{2}\left(h \frac{\partial^{2} \zeta_{n}}{\partial X^{2}}-\frac{\partial^{2} \zeta_{n}}{\partial T^{2}}\right)+\mu\left(2 i n \sqrt{h}+\mu \frac{\partial h}{\partial X}\right) \frac{\partial \zeta_{n}}{\partial X}
$$




$$
\begin{aligned}
& +\quad \mu\left(2 i n+\mu^{2} \frac{4 i n^{3} h}{3}\right) \frac{\partial \zeta_{n}}{\partial T}+\left(\mu \frac{i n}{2 \sqrt{h}} \frac{\partial h}{\partial X}+\mu^{2} \frac{n^{4} h}{3}\right) \zeta_{n} \\
& +\quad \epsilon \frac{i n}{\sqrt{h}}\left[2 \sqrt{h}\left(\mu \frac{\partial \zeta_{n}}{\partial X}+\frac{i n}{2 \sqrt{h}} \zeta_{n}\right) u_{0}+\mu \frac{\partial}{\partial X}\left(\zeta_{0} \zeta_{n}\right)-\frac{2}{\sqrt{h}}\left(\mu \frac{\partial \zeta_{0}}{\partial T}-\frac{i n}{4} \zeta_{0}\right) \zeta_{n}\right] \\
& =\quad \epsilon \frac{3 n}{4 h} \sum_{s \neq 0, n}\left[n \zeta_{\diamond} \zeta_{n-s}+\mu 2 i \frac{\partial}{\partial T}\left(\zeta_{\diamond} \zeta_{n-s}\right)\right]+O\left(\epsilon^{2}, \epsilon \mu^{2}, \mu^{4}\right)
\end{aligned}
$$

The following relationships have been employed in deriving equation (11):

$$
\begin{gathered}
u_{n}=-\frac{i}{n \sqrt{h}}\left(i n+\mu \sqrt{h} \frac{\partial}{\partial X}+\mu \frac{\partial}{\partial T}\right) \zeta_{n}+O\left(\epsilon, \mu^{2}\right) \\
\left(i n+\mu \sqrt{h} \frac{\partial}{\partial X}\right) u_{n}=\frac{1}{\sqrt{h}}\left(i n-\mu \frac{\partial}{\partial T}\right) \zeta_{n}+O\left(\epsilon, \mu^{2}\right)
\end{gathered}
$$

In the long wave equations (9) and (10) the leading order terms, $O(\mu)$, indicate that free long waves can propagate in both positive and negative $x$-direction. The righthand side terms in the long wave equations provide the forcing through nonlinearity to generate bound long waves. On the other hand, the governing equation for short waves, (11), suggests that the leading order solution, $O(\mu)$, represents progressive waves propagating in the positive $x$-direction.

\section{Evolution Equations for Wave Envelopes}

In this section we decompose the wave envelope in both time and space in Fourier series. Assuming that the fundamental frequency of a wave group, $\mu \Omega \ll 1$, is known, higher harmonics are generated through nonlinearity. Hence the wave envelope, $\zeta_{n}$, and the velocity, $u_{\circ}$, can be expressed as

$$
\zeta_{n}=\sum_{m} \Psi_{n}^{m}(X) e^{-i m n T}
$$




$$
u_{\circ}=\sum_{m} U_{0}^{m}(X) e^{-i m \Omega T}
$$

where $m=0, \pm 1, \pm 2, \ldots$. By substituting equations (14) and (15) into equations (9)(11) and after lengthy algebra a set of governing equations for $\Psi_{0}^{m}$ (for infragravity waves) and $\Psi_{n}^{m}$ ( $n \neq 0$ for short waves) can be derived as:

$$
\begin{gathered}
\mu^{2}\left[\frac{d}{d X}\left(h \frac{d \Psi_{o}^{m}}{d X}\right)+m^{2} \Omega^{2} \Psi_{\circ}^{m}\right]=-\frac{\epsilon \mu^{2}}{2} \sum_{p \neq 0, m} \frac{m}{m-p} \frac{d}{d X}\left(\Psi_{\circ}^{p} \frac{d \Psi_{o}^{m-p}}{d X}\right) \\
+\quad \frac{\epsilon \mu^{2}}{4} \sum_{p \neq 0, m} \frac{h}{p(m-p) \Omega^{2}} \frac{d^{2}}{d X^{2}}\left(\frac{d \Psi_{o}^{p}}{d X} \frac{d \Psi_{\circ}^{m-p}}{d X}\right)-\frac{\epsilon \mu^{2}}{4} \sum_{s \neq 0} \sum_{p} \frac{d^{2}}{d X^{2}}\left(\Psi_{s}^{p} \Psi_{-s}^{m-p}\right) \\
-\quad \frac{\epsilon \mu^{2}}{2} \sum_{s \neq 0} \sum_{p} \frac{i m \Omega}{\sqrt{h}} \frac{d}{d X}\left(\Psi_{s}^{p} \Psi_{-s}^{m-p}\right)+O\left(\epsilon^{2} \mu, \epsilon \mu^{3}, \mu^{5}\right)
\end{gathered}
$$

in which $p=0, \pm 1, \pm 2, \ldots$ and $s=0, \pm 1, \pm 2, \ldots$. As mentioned in the previous section, the homogeneous part of equation (16) suggests that free long wave components propagate in both $\pm x$-direction with a phase speed $\sqrt{h}$, while the inhomogeneous solutions correspond to the bound long waves. For short waves $(n \neq 0)$ the governing equation becomes

$$
\begin{aligned}
& \mu^{2} \frac{d}{d X}\left(h \frac{d \Psi_{n}^{m}}{d X}\right)+\mu 2 i n \sqrt{h} \frac{d \Psi_{n}^{m}}{d X} \\
+\quad & {\left[\mu \frac{i n}{2 \sqrt{h}} \frac{d h}{d X}+\mu^{2} \frac{n^{3} h}{3}(n+4 m \mu \Omega)+m \mu \Omega(2 n+m \mu \Omega)\right] \Psi_{n}^{m} } \\
= & \quad \epsilon \mu \frac{i n}{\sqrt{h}} \sum_{p \neq 0}\left[\frac{d}{d X}\left(\Psi_{0}^{p} \Psi_{n}^{m-p}\right)-\frac{2 i \sqrt{h}}{p \mu \Omega}\left(\mu \frac{d \Psi_{n}^{m-p}}{d X}+\frac{i n}{2 \sqrt{h}} \Psi_{n}^{m-p}\right) \frac{d \Psi_{0}^{p}}{d X}\right] \\
+\quad & \epsilon \frac{n}{2 h} \sum_{p \neq 0}(n+4 p \mu \Omega) \Psi_{0}^{p} \Psi_{n}^{m-p}+\epsilon \frac{3 n(n+2 m \mu \Omega)}{4 h} \sum_{s \neq 0, n} \sum_{p} \Psi_{s}^{p} \Psi_{n-s}^{m-p} \\
+\quad & O\left(\epsilon^{2}, \epsilon \mu^{2}, \mu^{4}\right)
\end{aligned}
$$

It is remarked here that long waves, $\Psi_{0}^{p}$, appear in the right-hand side of the above equation. Therefore, short waves and long waves are fully coupled. 
A splitting scheme is used to separate the right- and left-going waves (Kirby 1986). Let $\Psi_{\circ}^{m}$ be the sum of right- and left-going long waves:

$$
\Psi_{0}^{m}=\Psi_{0}^{m_{+}}+\Psi_{0}^{m-}
$$

We then assume the coupled equations

$$
\begin{gathered}
\frac{d \Psi_{\circ}^{m_{+}}}{d X}=\frac{i m \Omega}{\sqrt{h}} \Psi_{\circ}^{m_{+}}+H_{m} \\
\frac{d \Psi_{\circ}^{m_{-}}}{d X}=-\frac{i m \Omega}{\sqrt{h}} \Psi_{0}^{m_{-}}-H_{m}
\end{gathered}
$$

where the coupling term $H_{m}$ is to be determined. Substituting equations (18)-(20) into equation (16), we obtain

$$
\begin{aligned}
& H_{m}=-\frac{1}{4 h} \frac{d h}{d X}\left(\Psi_{\circ}^{m_{+}}-\Psi_{\circ}^{m}-\right)-\epsilon \frac{i 3 m \Omega}{8 h \sqrt{h}} \sum_{p \neq 0, m}\left(\Psi_{\circ}^{p_{+}} \Psi_{\circ}^{(m-p)_{+}}+\Psi_{\circ}^{p-} \Psi_{\circ}^{(m-p)-}\right) \\
& -\epsilon \frac{i \Omega}{8 m h \sqrt{h}} \sum_{p \neq 0, m}\left(m^{2}-4 p^{2}\right)\left(\Psi_{o}^{p+} \Psi_{o}^{(m-p)}-+\Psi_{o}^{p-} \Psi_{o}^{(m-p)_{+}}\right) \\
& +\epsilon \frac{i}{8 m \Omega \sqrt{h}} \sum_{s \neq 0} \sum_{p}\left[\frac{d^{2}}{d X^{2}}\left(\Psi_{s}^{p} \Psi_{-s}^{m-p}\right)+\frac{2 i m \Omega}{\sqrt{h}} \frac{d}{d X}\left(\Psi_{s}^{p} \Psi_{-s}^{m-p}\right)\right] \\
& +O\left(\epsilon^{2}, \epsilon \mu^{2}, \mu^{4}\right)
\end{aligned}
$$

The amplitudes of the wave envelope evolve because of nonlinearity, shoaling and reflection. Because the length scale for the bottom variation is longer than the wavelength of the short waves, the reflection of short waves is negligible. However, the reflection of wave group might be significant. The long waves can be expressed as:

$$
\Psi_{\circ}^{m+}=A_{\circ}^{m}(\bar{X}) e^{i m \odot}
$$




$$
\Psi_{\circ}^{m-}=B_{\circ}^{m}(\bar{X}) e^{-i m \Theta}
$$

in which

$$
\Theta=\Omega \int \frac{1}{\sqrt{h}} d X
$$

has been used and the slow variable $\widetilde{X}$ representing the evolution of the wave envelope is defined as:

$$
\bar{X}=\mu^{2} X
$$

The carrier waves can also be expressed in the similar form.

$$
\Psi_{n}^{m}=A_{n}^{m}(\widetilde{X}) e^{i m \Theta}
$$

Substituting equations (22), (23) and (25) into equations (16) and (17), we obtain a set of governing equations for long waves and short waves:

$$
\begin{aligned}
& \mu^{3} \frac{d A_{\circ}^{m}}{d \widetilde{X}}+\mu \frac{1}{4 h} \frac{d h}{d X} A_{\circ}^{m}-\mu \frac{1}{4 h} \frac{d h}{d X} e^{-i 2 m \odot} B_{\circ}^{m} \\
= & \quad \epsilon \frac{3 i m \mu \Omega}{8 h \sqrt{h}}\left(\sum_{p \neq 0, m} A_{\circ}^{p} A_{\circ}^{m-p}+\sum_{s \neq 0} \sum_{p} A_{s}^{p} A_{-s}^{m-p}\right)-\epsilon \frac{3 i m \mu \Omega}{8 h \sqrt{h}} \sum_{p \neq 0, m} B_{\circ}^{p} B_{\circ}^{m-p} e^{-i 2 m \Theta} \\
-\quad & \epsilon \frac{i \mu \Omega}{8 h \sqrt{h}} \sum_{p \neq 0, m} \frac{m^{2}-4 p^{2}}{m}\left(B_{\circ}^{p} A_{\circ}^{m-p} e^{-i 2 p \Theta}+A_{\circ}^{p} B_{\circ}^{m-p} e^{-i 2(m-p) \Theta}\right) \\
+\quad & O\left(\epsilon^{2}, \epsilon \mu^{2}, \mu^{4}\right)
\end{aligned}
$$

for right-going long waves,

$$
\begin{aligned}
& \mu^{3} \frac{d B_{\circ}^{m}}{d \bar{X}}+\mu \frac{1}{4 h} \frac{d h}{d X} B_{\circ}^{m}-\mu \frac{1}{4 h} \frac{d h}{d X} e^{+i 2 m \Theta} A_{\circ}^{m} \\
= & \epsilon \frac{3 i m \mu \Omega}{8 h \sqrt{h}} \sum_{p \neq 0, m} B_{\circ}^{p} B_{\circ}^{m-p}+\epsilon \frac{3 i m \mu \Omega}{8 h \sqrt{h}}\left(\sum_{p \neq 0, m} A_{\circ}^{p} A_{\circ}^{m-p}+\sum_{s \neq 0} \sum_{p} A_{s}^{p} A_{-\bullet}^{m-p}\right) e^{+i 2 m \odot}
\end{aligned}
$$




$$
\begin{aligned}
& +\quad \epsilon \frac{i \mu \Omega}{8 h \sqrt{h}} \sum_{p \neq 0, m} \frac{m^{2}-4 p^{2}}{m}\left(A_{\circ}^{p} B_{\circ}^{m-p} e^{+i 2 p \odot}+B_{\circ}^{p} A_{\circ}^{m-p} e^{+i 2(m-p) \odot}\right) \\
& +\quad O\left(\epsilon^{2}, \epsilon \mu^{2}, \mu^{4}\right)
\end{aligned}
$$

for left-going long waves, and

$$
\begin{gathered}
\mu^{3} \frac{d A_{n}^{m}}{d \widetilde{X}}+\left[\mu \frac{1}{4 h} \frac{d h}{d X}-\mu^{2} \frac{i n^{3} \sqrt{h}}{6}\left(1+\frac{4 m \mu \Omega}{n}\right)\right] A_{n}^{m} \\
=-\quad \epsilon \frac{i(n+2 m \mu \Omega)}{8 h \sqrt{h}}\left(\sum_{p \neq 0} 6 A_{o}^{p} A_{n}^{m-p}+\sum_{s \neq 0, n} \sum_{p} A_{s}^{p} A_{n-s}^{m-p}\right) \\
+\quad \epsilon \frac{i}{4 h \sqrt{h}} \sum_{p \neq 0}[n+2(m-2 p) \mu \Omega] B_{0}^{p} A_{n}^{m-p} e^{-i 2 p \odot}+O\left(\epsilon^{2}, \epsilon \mu^{2}, \mu^{4}\right)
\end{gathered}
$$

for short waves. In long wave equations (26) and (27), the second and third terms on the left-hand side denote shoaling and reflection effects, respectively, while the right-hand side terms represent nonlinear interactions, which include self interactions and cross interactions among left-going and right-going long waves, and interactions among different harmonics of short waves.

Once $A_{\circ}^{m}, B_{\circ}^{m}$, and $A_{n}^{m}$ are obtained, the free-surface displacement for long waves and wave groups can be recovered from equations (6), (14), (22) and (23); i.e.

$$
\zeta=\sum_{m} A_{o}^{m} \exp \left[i m \Omega\left(\int \frac{1}{\sqrt{h}} d X-T\right)\right]
$$

for right-going long waves,

$$
\zeta=\sum_{m} B_{o}^{m} \exp \left[i m \Omega\left(-\int \frac{1}{\sqrt{h}} d X-T\right)\right]
$$

for left-going long waves, and

$$
\zeta=\frac{1}{2} \sum_{n} \sum_{m} A_{n}^{m} \exp \left[i m \Omega\left(\int \frac{1}{\sqrt{h}} d X-T\right)\right] \exp \left[i n\left(\int \frac{1}{\sqrt{h}} d x-t\right)\right]
$$

for wave groups. 


\section{Numerical Examples}

In this section numerical results are presented for the propagation and reflection of wave groups and long waves by slowly varying bottom topography. The coupled nonlinear governing equations for wave groups and carrier waves derived in the previous section are integrated with the fourth-order Runge-Kutta method. The iteration technique developed by Liu and Tsay (1983) is used to solve the coupled equations for weak reflection problems. In this iterative scheme, the transmitted long wave field is first solved without the consideration of a reflected long wave field. The reflected long wave is then calculated with the transmitted wave solutions. Finally, the transmitted long wave field is updated with the newly obtained reflected long wave field. The procedure is repeated until the converged solutions are obtained. In present study the convergence condition is defined as

$$
\frac{\left.|| A_{\circ}^{m}\right|^{k}-\left|A_{\circ}^{m}\right|^{k-1} \mid}{\left|A_{\circ}^{m}\right|^{k-1}}<10^{-4}, \quad \frac{\left.|| B_{\circ}^{m}\right|^{k}-\left|B_{\circ}^{m}\right|^{k-1} \mid}{\left|B_{\circ}^{m}\right|^{k-1}}<10^{-4}
$$

where $k-1$ and $k$ represent the previous and current iteration steps. Converged solutions are usually obtained after three or four iterations in the present study. In present numerical calculations, the following initial conditions are used for carrier waves throughout this study.

$$
A_{1}^{\circ}=1.0, \quad A_{1}^{1}=1.0
$$

i.e. the primary waves constitute a regular wave group and the corresponding normalized frequencies are 1 and $1+\mu \Omega$, respectively. 
Now we examine the Bragg resonance reflection for long waves over a periodic sandbar field with a finite length. The depth is expressed as

$$
h=1-\rho \sin (\delta x), \quad 0 \leq x \leq L
$$

in which $\rho$ is the dimensionless amplitude and $\delta$ is the wavenumber of the ripple bed. The orders of magnitude of $\rho$ and $\delta$ are $O(\epsilon)$ and $O(\mu)$, respectively. Rewriting the governing equations for long waves as

$$
\begin{aligned}
& \mu^{3} \frac{d A_{\circ}^{m}}{d \widetilde{X}}+\mu \frac{1}{4 h} \frac{d h}{d X} A_{\circ}^{m}-\mu \frac{1}{4 h} \frac{d h}{d X} e^{-i 2 m \Theta} B_{\circ}^{m}=N L T+O\left(\epsilon^{2}, \epsilon \mu^{2}, \mu^{4}\right) \\
& \mu^{3} \frac{d B_{\circ}^{m}}{d \widetilde{X}}+\mu \frac{1}{4 h} \frac{d h}{d X} B_{\circ}^{m}-\mu \frac{1}{4 h} \frac{d h}{d X} e^{+i 2 m \Theta} A_{\circ}^{m}=N L T+O\left(\epsilon^{2}, \epsilon \mu^{\ddot{2}}, \mu^{4}\right)
\end{aligned}
$$

in which NLT represents nonlinear terms. Using equation (34) and

$$
\mu \frac{d h}{d X}=-\rho \delta \frac{e^{i \delta x}+e^{-i \delta x}}{2}
$$

in equations (35) and (36), we can express the third terms on the left-hand side of (35) and (36) in the following forms

$$
-\mu \frac{1}{4 h} e^{-i \beta} B_{\circ}^{m}, \quad-\mu \frac{1}{4 h} e^{i \beta} A_{\circ}^{m}
$$

where $\beta$ is written as

$$
\beta=2 m \mu \Omega \int \frac{1}{\sqrt{h}} d x-\delta x
$$

In general, $e^{ \pm i \beta}$ are fast varying terms. Therefore, the influence of the reflected long waves, $B_{\circ}^{m}$, on the slowly modulating transmitted long waves, $A_{\circ}^{m}$, is negligible, and 
vice versa. Consequently, the reflected long wave is insignificant. However, when $\beta$ is zero, i.e.

$$
\delta \approx 2 m \mu \Omega
$$

the fast varying components, $e^{ \pm i \beta}$, become unity. Hence the reflected long waves, $B_{\circ}^{m}$, are as important as the transmitted long waves, $A_{\circ}^{m}$. Equation (39) is the Bragg resonance reflection condition for long waves.

In the first example numerical results are obtained for $\rho=0.08$ and $\delta=0.222$. The ripple bed begins at $x=0$ and ends at $x=L=90 \pi$. Therefore, there are ten ripples within the ripple bed. Two sets of wave parameters have been employed: $\epsilon=0.02$, $\mu^{2}=0.09$ and $\epsilon=0.08, \mu^{2}=0.1$. In both cases, $n_{\max }=4$ and $m_{\max }=4$ are used and the fundamental frequency of the wave group $(\mu \Omega)$ is 0.111 which satisfies the resonance reflection condition for the first harmonic $(m=1)$, (39). In figure 2 snap shots of free surface profiles and the transmitted long waves (first harmonic only) over the ripple bed are presented. Because the nonlinearity is much stronger in the second case (figure 2(b)) than in the first case (figure 2(a)), the amplitude of the transmitted long waves is much higher and the free surface profile eventually becomes irregular. In figures 3 and 4 four harmonics of both transmitted and reflected long waves are plotted over the ripple bed. In both cases, the first harmonic $(m=1)$ dominates in the reflected long waves because of resonance, while higher harmonics become more important when the nonlinearity is significant (see figure 4 (a)). Figure 5 shows the amplitude spectrum of the wave group at the end of sandbar field, $x=L$. For the case 
with stronger nonlinearity more energy is transferred to the other harmonics from the initial conditions, $A_{1}^{\circ}$ and $A_{1}^{1}$.

Next we investigate the resonance reflection with a variable length of the rippled bed, $L$, but with a fixed number of ripples, $N$. We use two sets of different lengths for the ripple bed: $L=9 \lambda(N=9), \lambda$ is the wavelength of a ripple, and $L=18 \lambda(N=18)$. Thus, choosing $\delta$ to vary from 0.2 to $1.2, L$ varies from $90 \pi$ to $15 \pi$ for $L=9 \lambda$, while $L$ varies from $180 \pi$ to $30 \pi$ for $L=18 \lambda$. Figure 6 shows the amplitudes of reflected long waves for each harmonic. The number of ripples is fixed as 18 . There are three resonant wavenumbers corresponding to $\delta=0.4(m=1), 0.8(m=2)$ and $1.2(m=3)$. The amplitude of the first harmonic is much larger than those of the second and the third because the relative importance of higher harmonics decreases as $m$ increases. Hence, the first resonance amplitude is also much bigger than the others. Figure 7 shows a set of amplitudes of reflected long waves for two different values of $N ; N=9$ and 18. The resonance occurs at $\delta=0.4,0.8$ and 1.2 for both cases. The magnitude of resonant amplitudes of $N=18$ case is much larger than that of $N=9$ case. That is, the resonance effect can be amplified by the increase of the number of ripples (Kirby and Vengayil 1988).

Finally we examine the resonance reflection problem with a fixed length of ripple bed, $L$. Thus the wavelength of a ripple and the number of ripples vary as the wavenumber of a ripple varies. Figure 8 shows a set of amplitudes of reflected long waves for three different values for $L ; L=30 \pi, 45 \pi$ and $60 \pi$. The resonance occurs at 
$\delta=0.4$ and 0.8 for all cases. The corresponding numbers of ripples are $N=6(\delta=0.4)$ and $12(\delta=0.8)$ for $L=30 \pi, 9$ and 18 for $L=45 \pi$, and 12 and 24 for $L=60 \pi$. The magnitude of resonant amplitude increases as the number of ripples increases.

\section{Conclusions}

In this paper we have derived a set of governing equations from Boussinesq equations to study the evolution of wave groups and long waves in a slowly varying depth in shallow water. A simple splitting technique is employed to get the coupled nonlinear evolution equations for right- and left-going long waves.

The evolution of wave groups and long waves are examined over the sinusoidally varying bottom topography. The reflected long waves could be resonated by the interaction with bottom topography under the Bragg reflection condition. The magnitude of resonant effect is not surprising but appreciable.

In this study we show only the numerical results of propagation and reflection of wave group and long waves due to the lack of field or experimental data. The comparison of numerical result to the field data would be valuable to understand the formation mechanism of longer-spaced multiple sandbars. 


\section{Acknowledgements}

This research has been supported by a research grant from the Army Research Office (DAAL 03-92-G-0116) and a research project from the New York Sea Grant Institute. 


\section{References}

1. Bagnold, R. A., 1946, "Motion of waves in shallow water: interaction of waves and sand bottom", Proc. R. Soc. London, 87, pp. 1-15.

2. Carter, T. G., Liu, P. L.-F. and Mei, C. C., 1973, "Mass transport by waves and offshore sand bedforms", J. Waterways, Harbor and Coastal Engrg. Div., $A S C E, 99$, pp. 165-184.

3. Davies, A. G. and Heathershaw A. D., 1984, "Surface wave propagation over sinusoidally varying topography", J. Fluid Mech., 144, pp. 419-443.

4. Elgar, S., Herbers, T. H. C., Okihiro, M., Oltman-Shay, J. and Guza, R. T., 1992, "Observations of infragravity waves", J. Geophysical Research, 97, pp. $15573-15577$.

5. Hara, T. and Mei, C. C., 1987, "Bragg reflection of surface waves by periodic bars: theory and experiment", J. Fluid Mech., 178, pp. 221-241.

6. Katoh, K., 1984, "Multiple longshore bars formed by long period standing waves", Report of the Port and Harbor Research Institute, 23, pp. , (in Japanese).

7. Kirby, J. T., 1986, "A general wave equation for waves over rippled beds", J. Fluid Mech., 162, pp. 171-186.

8. Kirby, J. T. and Vengayil, P., 1988, "Nonresonant and resonant reflection of long waves in varying channels", J. Geophysical Research, 93, pp. 10782-10796. 
9. Liu, P. L.-F. and Tsay, T.-K., 1983, "On weak reflection of water waves", J. Fluid Mech.. 131, pp. 59-71.

10. Liu, P. L.-F., Yoon, S. B. and Cho, Y.-S., 1992, "Shoaling of wave groups in shallow water", in Nonlinear Dispersive Wave Systems, ed. by L. Debnath, pp. 41-56, World Scientific.

11. Liu, P. L.-F., Yoon, S. B. and Kirby, J. T., 1985, "Nonlinear refraction-diffraction of waves in shallow water", J. Fluid Mech.. 153, pp. 184-201.

12. Mei, C. C., 1985, "Resonant reflection of surface water waves by periodic bars", J. Fluid Mech., 152, pp. 315-335.

13. Mei, C. C. and Liu, P. L.-F., 1993, "Surface waves and coastal dynamics", Annu. Rev. Fluid Mech., 25, pp. 215-240.

14. Peregrine, D. H., 1972, "Equations for water waves and the approximation behind them", in Waves and Beaches, ed. by R. E. Meyer, pp. 95-121, Academic Press.

15. Short, A. D., 1975, "Multiple offshore bars and standing waves", J. Geophysical Research, 80, pp. 3838-3840.

16. Yoon, S. B. and Liu, P. L.-F., 1987, "Resonant reflection of shallow-water waves due to corrugated boundaries", J. Fluid Mech., 180, pp. 451-469. 


\section{$\underline{\text { Figure Captions }}$}

Figure 1. An aerial photograph of multiple offshore bars off the northwestern Danish coast.

Figure 2. The evolution of wave groups (-) and transmitted long waves $(m=1, \ldots$ - ) ) over the rippled bed: (a) $\epsilon=0.02, \mu^{2}=0.09$, (b) $\epsilon=0.08, \mu^{2}=0.1(\mu \Omega=0.111$, $\left.\rho=0.08, \delta=0.222, x_{1}=0, x_{2}=90 \pi, n=4, m=4\right)$.

Figure 3. The evolution of long waves over a fixed length of the rippled bed: (a) transmitted long waves, (b) reflected long waves $\left(\epsilon=0.02, \mu^{2}=0.09, \mu \Omega=0.111\right.$, $\left.\rho=0.08, \delta=0.222, x_{1}=0, x_{2}=90 \pi, n=4, m=4\right)$.

Figure 4. The evolution of long waves over a fixed length of the rippled bed: (a) transmitited long waves, (b) reflected long waves $\left(\epsilon=0.08, \mu^{2}=0.1, \mu \Omega=0.111\right.$, $\left.\rho=0.08, \delta=0.222, x_{1}=0, x_{2}=90 \pi, n=4, m=4\right)$.

Figure 5. The evolution of amplitude spectrum of the wave group at the end the rippled bed, $\mathrm{x}=x_{2}: \circ \circ \circ ; \epsilon=0.02, \mu^{2}=0.09, \cdots ; \epsilon=0.08, \mu^{2}=0.1(\mu \Omega=0.111$, $\left.\rho=0.08, \delta=0.222, x_{1}=0, x_{2}=90 \pi, n=4, m=4\right)$.

Figure 6. The amplitudes of reflected long waves for each harmonic over a fixed number of ripples $(N=18)$ at $x_{1}=0:-; m=1, \ldots ; m=2, \ldots ; m=3, \ldots$ - ; $m=4\left(\epsilon=0.02, \mu^{2}=0.08, \mu \Omega=0.2, \rho=0.08, n=4, m=4\right)$. 
Figure 7. The amplitudes of reflected long waves over a fixed number of ripples at

$$
\begin{aligned}
& x_{1}=0: \ldots ; N=9(L=9 \lambda),-; N=18(L=18 \lambda)\left(\epsilon=0.02, \mu^{2}=0.08, \mu \Omega=0.2,\right. \\
& \rho=0.08, n=4, m=4) .
\end{aligned}
$$

Figure 8. The amplitudes of reflected long waves over a fixed length of the rippled bed at $x_{1}=0:-\cdots ; x_{2}=30 \pi, \ldots ; x_{2}=45 \pi, \longrightarrow ; x_{2}=60 \pi\left(\epsilon=0.02, \mu^{2}=0.08\right.$, $\mu \Omega=0.2, \rho=0.08, n=4, m=2)$. 


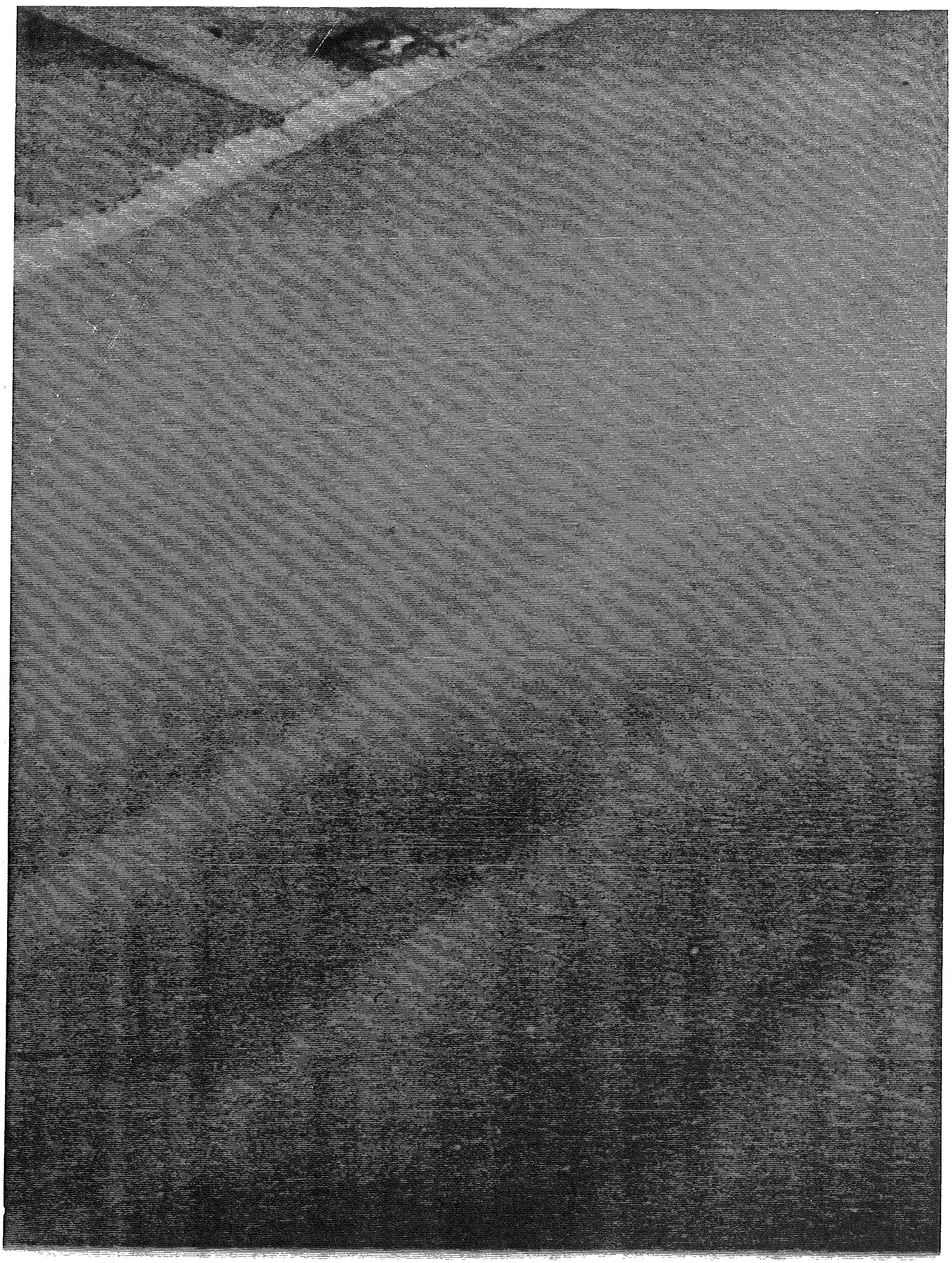




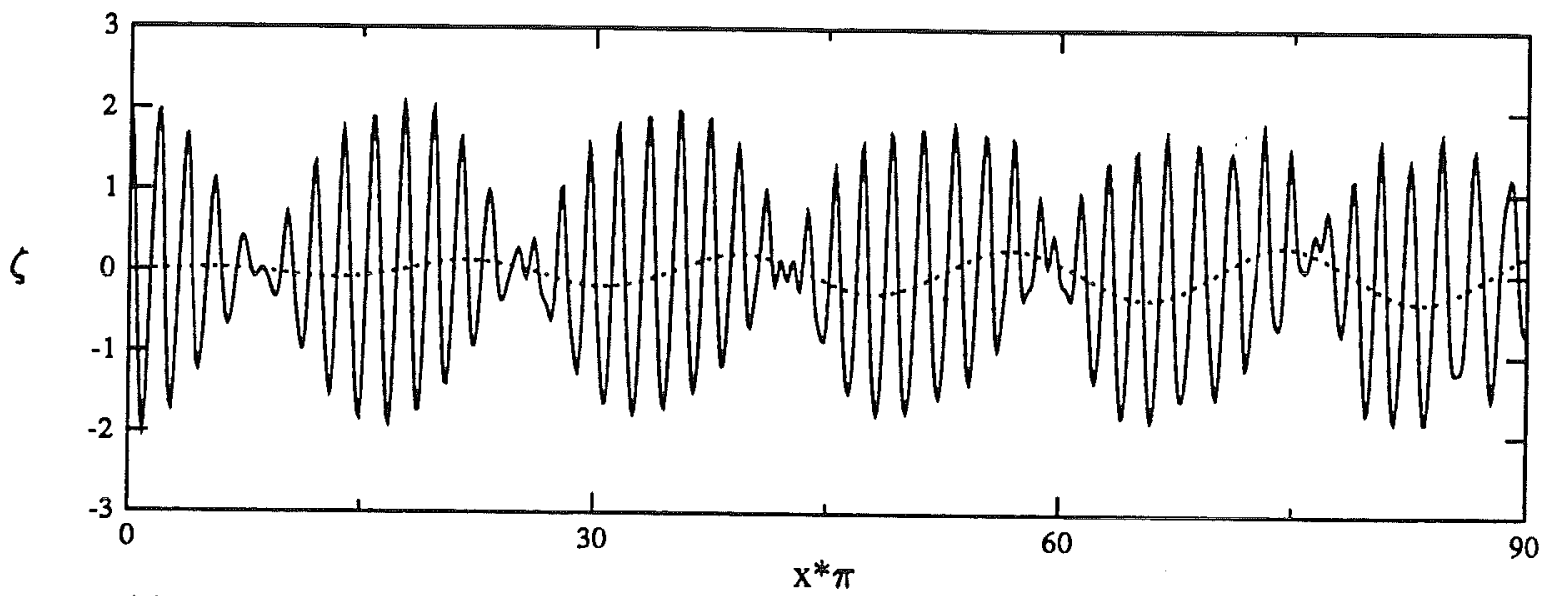

(a)

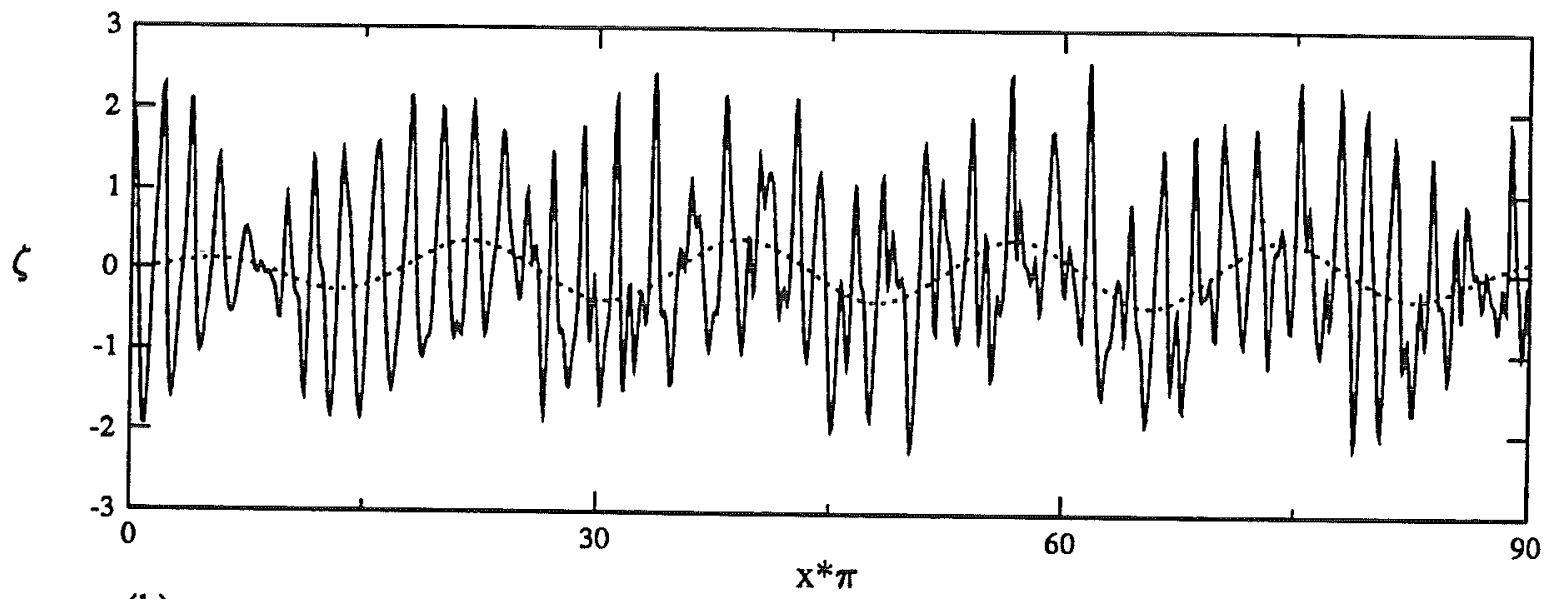

(b)

Figure 2. 


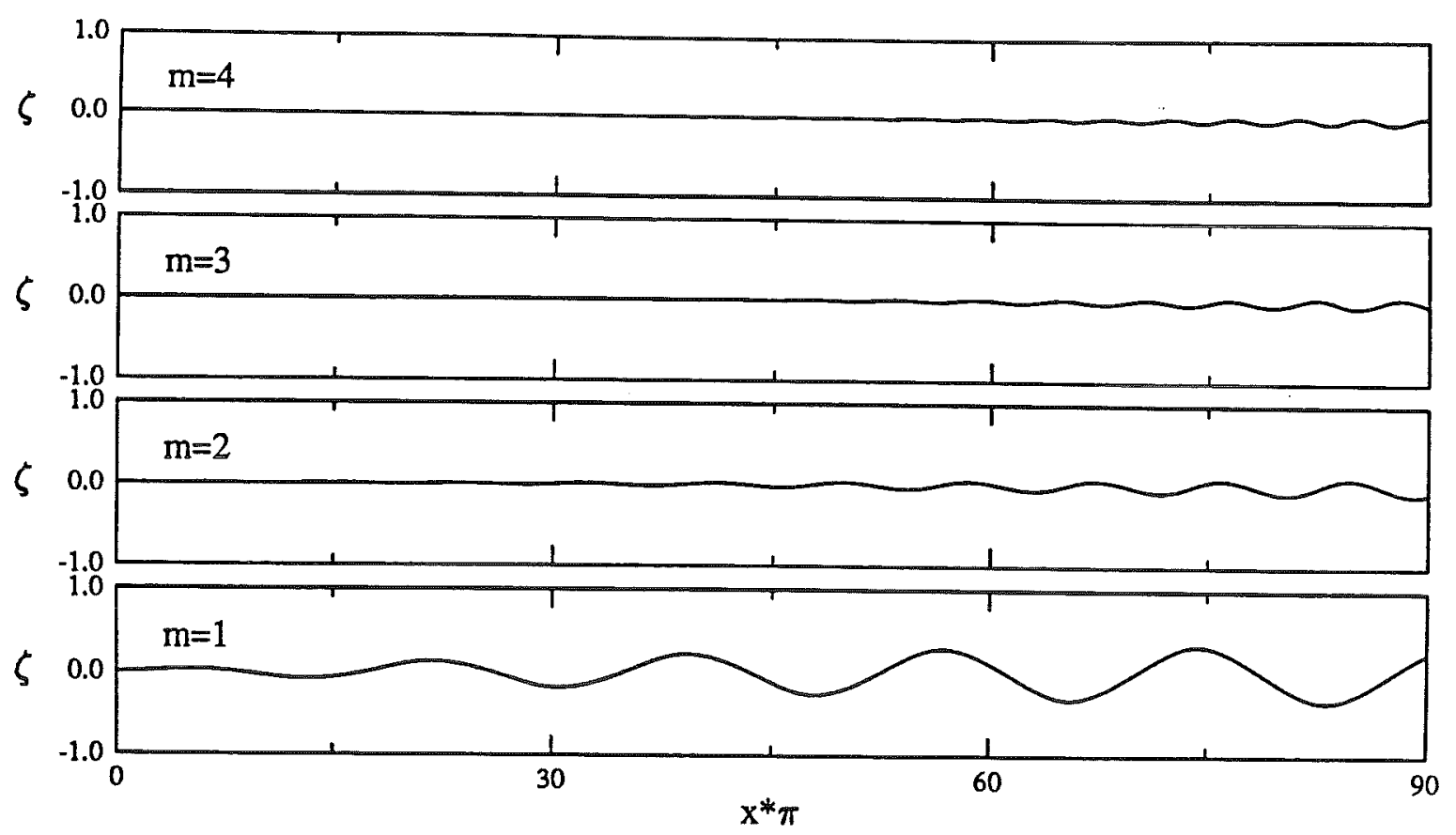

(a)
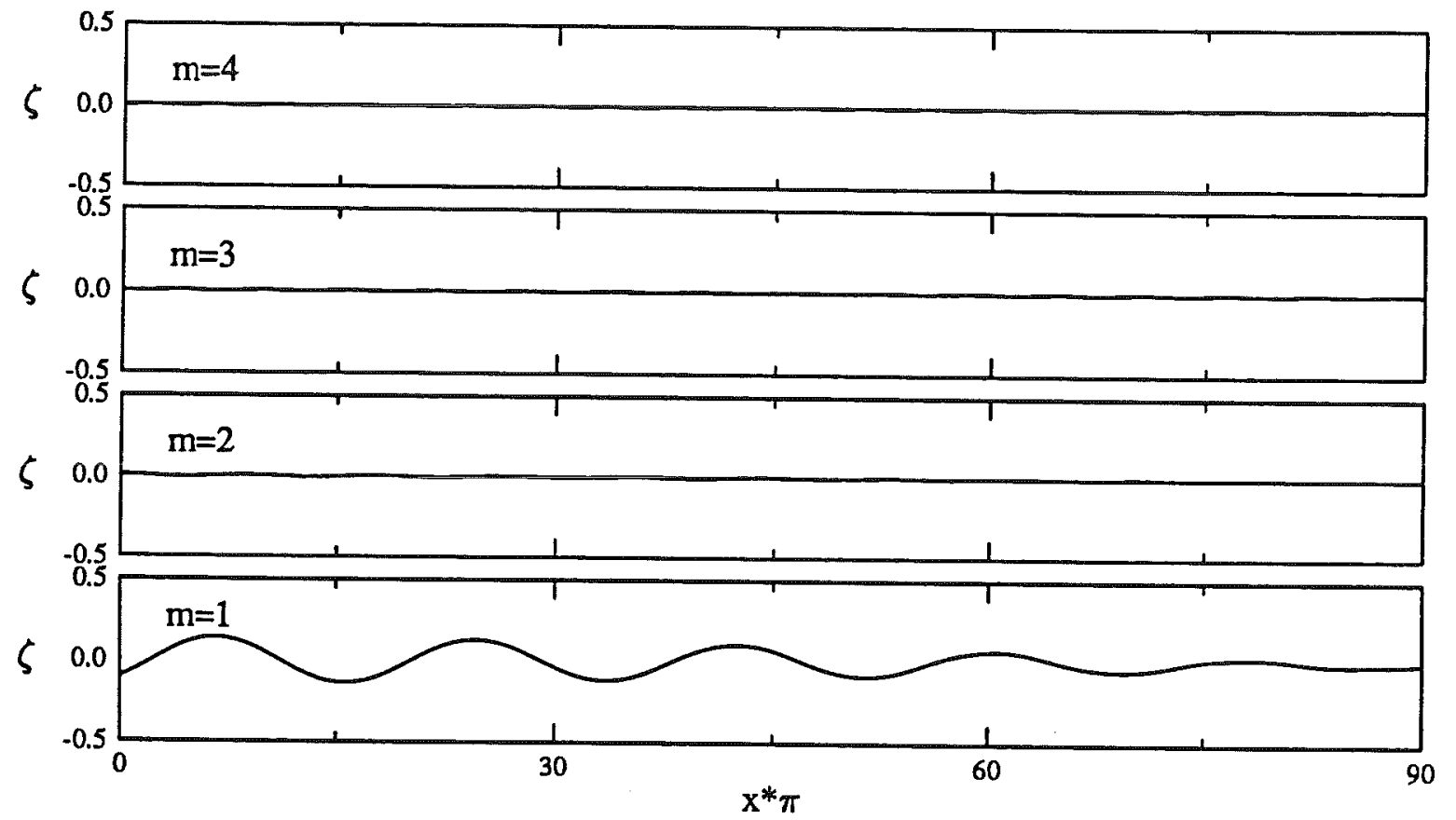

(b)

Figure 3. 


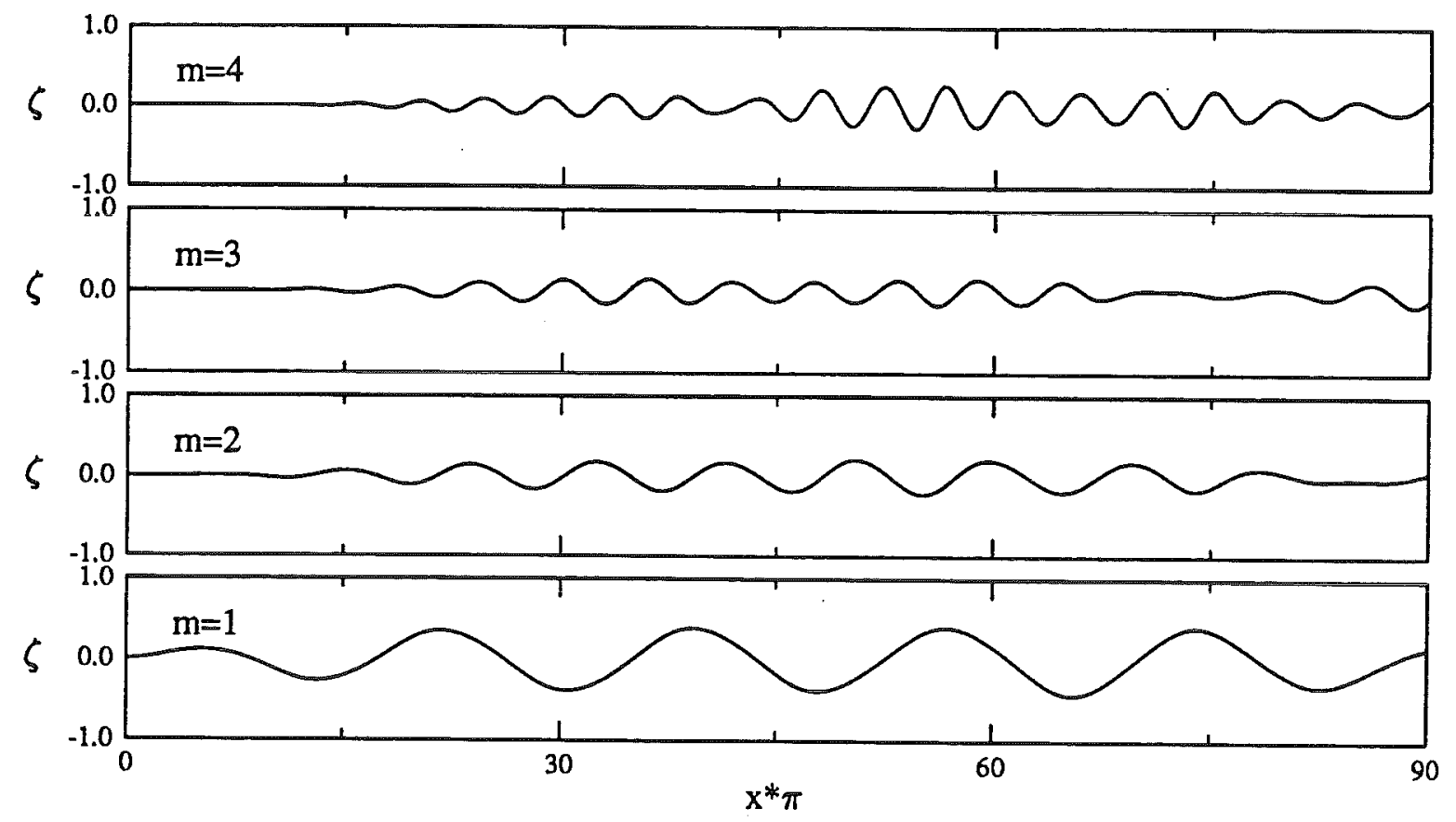

(a)

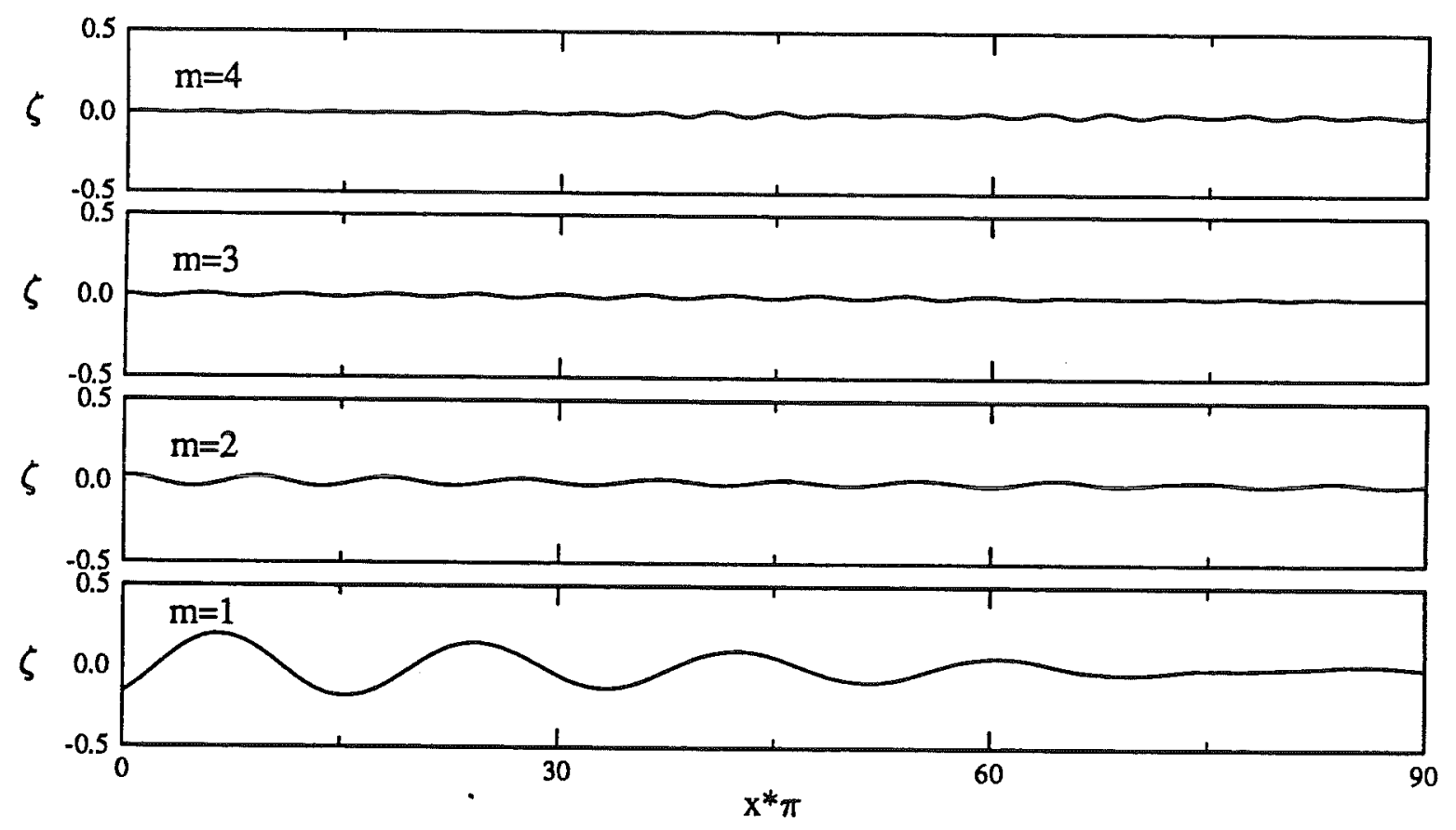

(b)

Figure 4. 


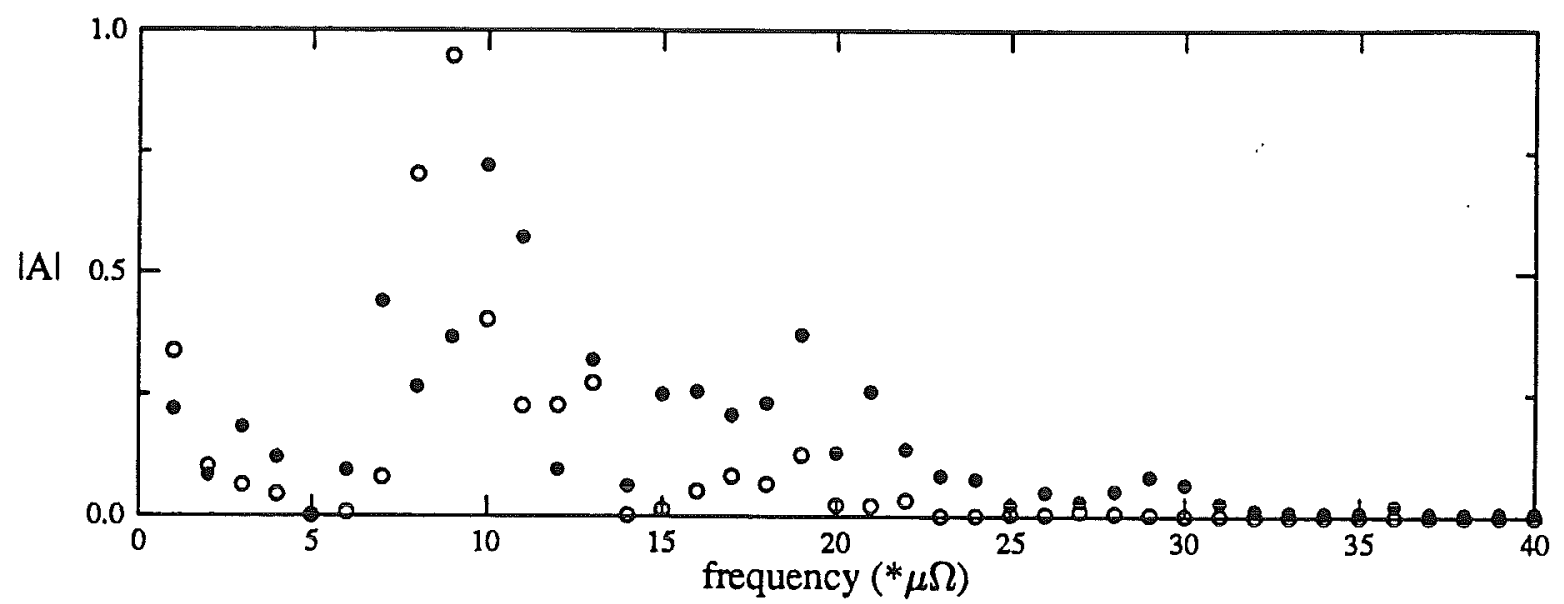

Figure 5. 


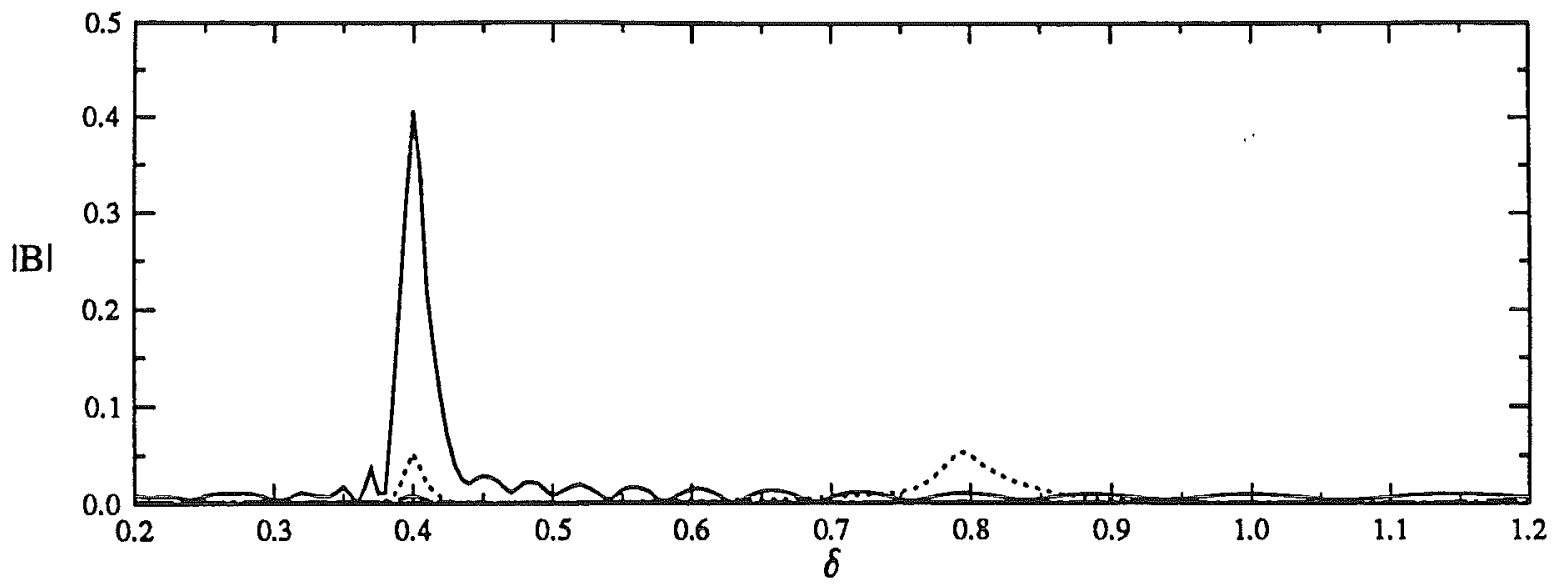

Figure 6. 


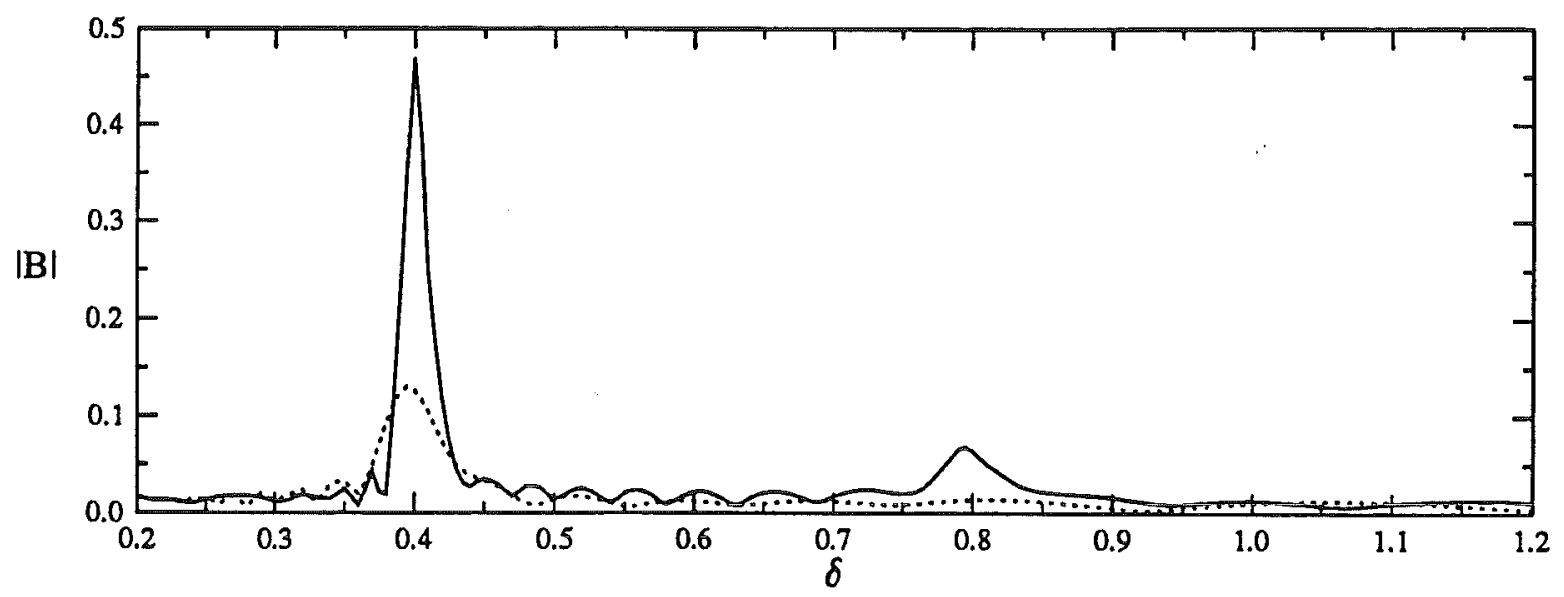

Figure 7. 


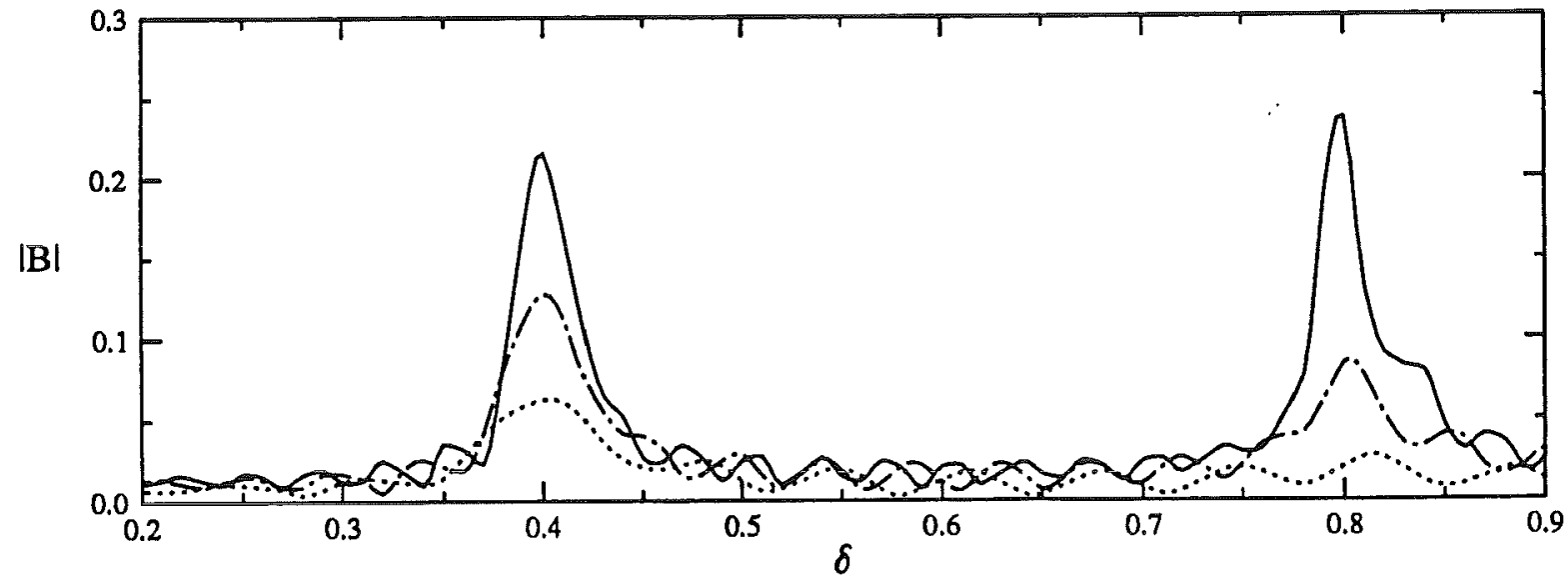

Figure 8. 